\title{
PREPARAÇÕES E APLICAÇÕES SINTÉTICAS RECENTES DE ENAMINONAS
}

Helena M. C. Ferraz* e Erika R. S. Gonçalo

Instituto de Química, Universidade de São Paulo, CP 26077, 05513-970 São Paulo - SP, Brasil

Recebido em 31/5/06; aceito em 16/8/06; publicado na web em 26/3/07

RECENT PREPARATIONS AND SYNTHETIC APPLICATIONS OF ENAMINONES. Enaminones are $\beta$-enamino carbonylic compounds bearing the conjugated system $\mathrm{N}-\mathrm{C}=\mathrm{C}-\mathrm{C}=\mathrm{O}$, which makes them versatile ambident synthetic building blocks, particularly in synthesis of heterocycles. This review covers the last three years of the literature concerning the preparation and synthetic applications of enaminones.

Keywords: enaminones; preparation and synthetic applications; N-heterocycles.

\section{INTRODUÇÃO}

Em um artigo anterior ${ }^{1}$, apresentamos uma revisão sobre os métodos de preparação de enaminonas, cobrindo a literatura no período de 1993 a 2001. Conforme mencionado ao final da citada revisão, pretendíamos apresentar uma segunda parte, versando sobre a reatividade destes compostos. Entretanto, devido à enorme importância e atualidade do tema, uma quantidade considerável de trabalhos dedicados à preparação e à aplicação sintética de $\beta$-enaminonas continua a aparecer freqüentemente na literatura. Nos últimos anos, algumas revisões interessantes e bastante gerais também foram publicadas, cobrindo a literatura até o início de 2003, conforme mencionado a seguir.

Kascheres et al. ${ }^{2}$ relatam os recentes avanços sobre preparação, reatividade e atividade biológica de enamino cetonas e enamino tionas, bem como sua utilização na preparação de compostos heterocíclicos. Elassar e El-Khair ${ }^{3}$ discutem alguns métodos de preparação e aplicações sintéticas das enaminonas. Outras duas revisões relatam a síntese de heterociclos a partir de 3-(dimetilamino)propeonatos ${ }^{4}$ e de enaminonas quirais ${ }^{5}$. Revisões anteriores cobrem a literatura até o início da década de $90^{6-8}$. Uma revisão mais específica, versando sobre pirróis, cita alguns exemplos de preparação destes a partir de enaminonas?.

Desta maneira, optamos por fazer, no presente artigo, uma atualização dos métodos de preparação, para em seguida apresentar as principais aplicações sintéticas desse versátil grupo de compostos, cobrindo a literatura a partir de 2003. Dos anos anteriores, apenas serão citadas as referências essenciais à compreensão do texto.

Apesar de já abordados na revisão anterior ${ }^{1}$, achamos conveniente reapresentar os principais aspectos da estrutura e da reatividade das $\beta$-enaminonas, de maneira bem resumida.

$\beta$-Enaminonas podem ser definidas como sendo compostos $\beta$ enamino carbonílicos - derivados de $\beta$-dicetonas, $\beta$-ceto ésteres e, por extensão, de outros análogos de compostos $\beta$-dicarbonílicos que apresentam o sistema conjugado $\mathrm{N}-\mathrm{C}=\mathrm{C}-\mathrm{C}=\mathrm{O}$. Os representantes mais comuns desta classe são as $\beta$-enamino cetonas (amidas vinílogas), os $\beta$-enamino ésteres (uretanas ou carbamatos vinílogos) e outras estruturas análogas ${ }^{10}$, como pode ser visto na Figura 1.

*e-mail: hmferraz@iq.usp.br

Dedicamos este artigo ao Prof. Dr. Sílvio do Desterro Cunha, da UFBA, pelo constante estímulo, esperando contribuir com mais uma "inevitável" leitura para seu grupo de pesquisa.

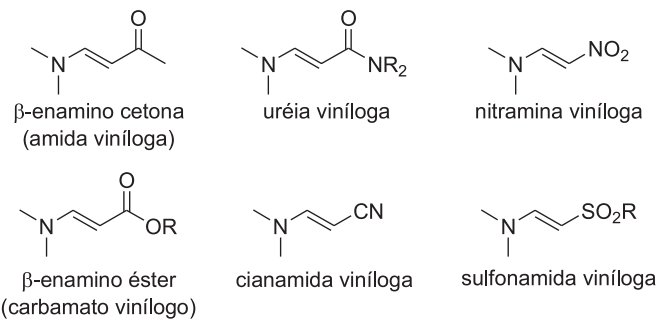

Figura 1. Os diversos tipos de $\beta$-enaminonas

As formas tautoméricas que as $\beta$-enaminonas podem adotar são enolimina (A), ceto-imina $(\mathbf{B})$ e ceto-enamina $(\mathbf{C})$. Na grande maioria dos casos, a forma predominante em que se encontram as $\beta$-enaminonas é a ceto-enamina $(\mathbf{C})$, que é estabilizada pela contribuição da forma polar $\mathbf{D}^{6}$ (Figura 2). A existência da forma $\mathbf{D}$ seria a provável explicação da formação de enaminonas em água, pois tal forma $\mathbf{D}$ pode ser solvatada pela água, inibindo a hidrólise da enaminona formada ${ }^{11}$.

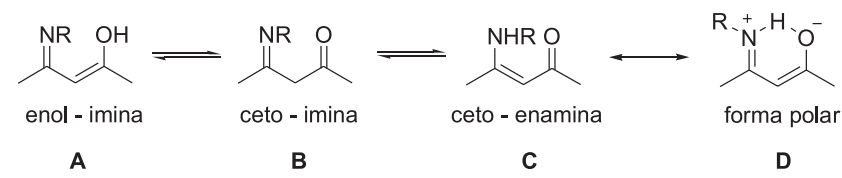

Figura 2. Formas tautoméricas das enaminonas

O tautomerismo imino-enamina pode favorecer o equilíbrio conformacional $^{12} \mathrm{e}$ as enaminonas podem adotar quatro possíveis conformações (Figura 3). Quando $\mathrm{R}^{1}=\mathrm{H}$, o confôrmero cis-s-cis pode ser estabilizado por ligação de hidrogênio intramolecular, sendo provavelmente o confôrmero de maior proporção ${ }^{3}$.

Tanto a reatividade como as propriedades físico-químicas das $\beta$-enaminonas estão relacionadas com a conformação e a distribui-

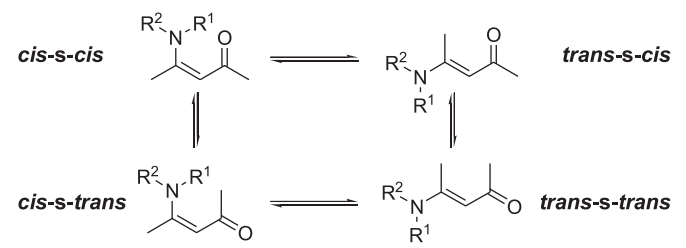

Figura 3. Conformações das enaminonas 
ção eletrônica do sistema $\mathrm{N}_{\mathrm{a}}-\mathrm{C}_{\mathrm{b}}=\mathrm{C}_{\mathrm{c}}-\mathrm{C}_{\mathrm{d}}=\mathrm{O}_{\mathrm{e}}$, que possui três centros nucleofílicos $(a-c-e)$ e dois eletrofílicos $(\beta-d)$, como pode ser visto na Figura 4. A possibilidade tanto de ataques eletrofílicos quanto nucleofílicos faz das $\beta$-enaminonas intermediários reacionais versáteis e com vasta aplicação sintética.

$$
\text { Centros Nucleofilicos : } \mathrm{N}_{2}-\mathrm{C}=\mathrm{C}_{c}-\mathrm{C}=\mathrm{O}_{e}
$$

Figura 4. Centros reativos das enaminonas

\section{MÉTODOS DE PREPARAÇÃo}

Neste item serão apresentados exclusivamente os métodos encontrados na literatura de 2003 em diante ou os que não constam de revisões ${ }^{1-9}$ anteriores.

Uma das maneiras mais usuais de classificar a preparação de enaminonas é através do tipo da reação empregada - como condensação, adição, acilação de enaminas e abertura de heterociclos. A preparação mais conhecida de $\beta$-enaminonas envolve a condensação direta de compostos $\beta$-dicarbonílicos com aminas, em refluxo com solventes aromáticos e remoção azeotrópica da água. Muitas adaptações nesse processo têm sido feitas, como o uso de suporte sólido, irradiação por microondas, ultra-som, líquido iônico e diversos catalisadores.

Em trabalho recente, Gholap et al. ${ }^{13}$ relatam a preparação de uma série de $\beta$-enaminonas, usando como catalisador cloreto de sílica para promover a reação sob condições heterogêneas e líquido iônico para as condições homogêneas. Uma variedade de substratos, incluindo acetato de amônio, aminas aromáticas e aminas alifáticas, foi condensada com compostos dicarbonílicos, à temperatura ambiente e com tempos reacionais baixos, em excelentes rendimentos. Exemplos ilustrativos encontram-se no Esquema 1.

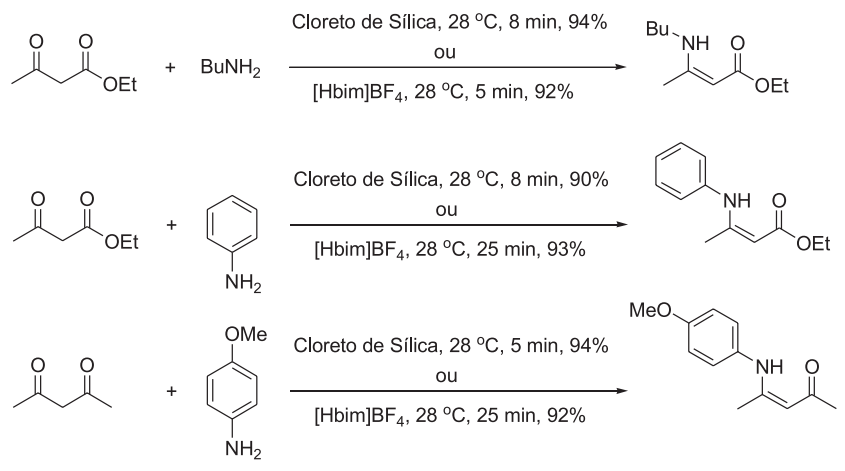

Esquema 1.

Dando continuidade a trabalhos anteriores, a irradiação por microondas (MO) foi utilizada por Braibante et al..$^{14}$ para obter uma série de $\beta$-enaminonas derivadas de $\alpha$-amino ésteres. As reações também foram efetuadas utilizando como suporte sólido K10 e KSF na presença de trietilamina, conforme exemplos mostrados no Esquema 2.

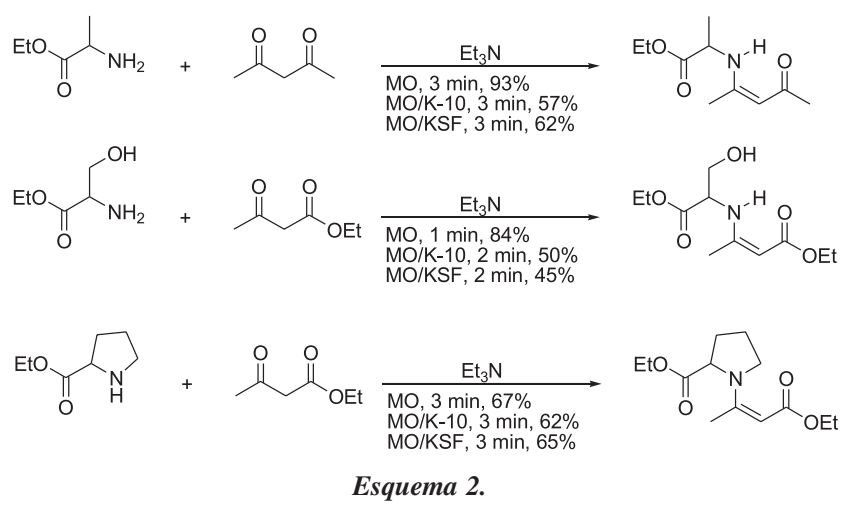

Dalpozzo et al. ${ }^{15}$ utilizaram triflato de érbio(III) como catalisador na preparação de aldiminas, cetiminas e enaminonas. Exemplos representativos encontram-se no Esquema 3. Um fato curioso é que o uso de solventes anidros ou de agentes secantes aumentou o tempo reacional e diminuiu o rendimento. Uma possível explicação seria de que somente cátions hidratados poderiam agir como ácidos de Lewis ${ }^{16}$. Assim, para a reação ser catalisada, seria necessária uma pequena quantidade de água para dissociar o triflato de érbio(III) e ativar o catalisador (Esquema 4).

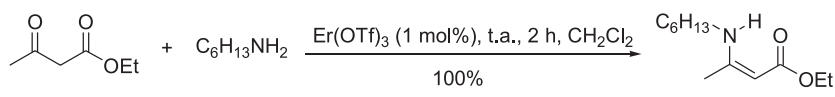

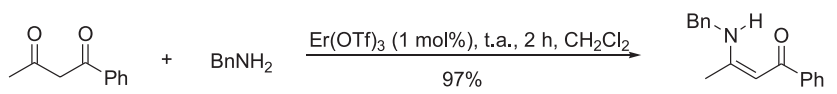

$$
\begin{aligned}
& +\mathrm{PhNH}_{2} \frac{\mathrm{Er}(\mathrm{OTf})_{3}(1 \mathrm{~mol} \%), \text { t.a., } 2,5 \mathrm{~h}, \mathrm{CH}_{2} \mathrm{Cl}_{2}}{95 \%}
\end{aligned}
$$

Esquema 3.

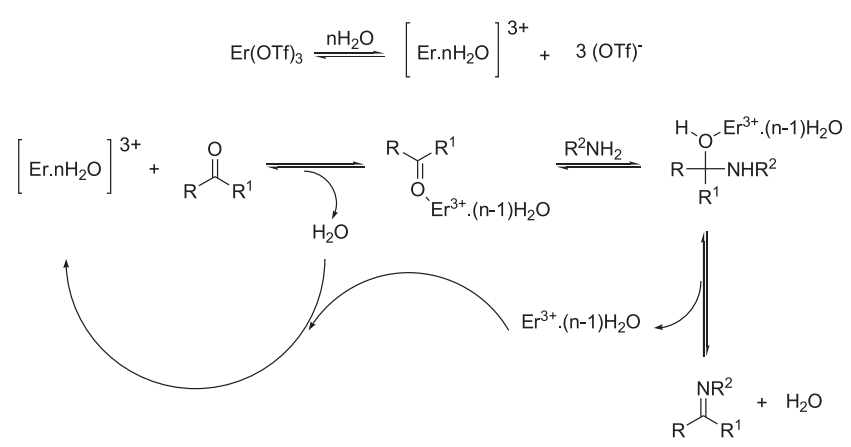

Esquema 4.

Bartoli et al. ${ }^{17}$ empregaram o perclorato de zinco $\left[\mathrm{Zn}\left(\mathrm{ClO}_{4}\right)_{2} \cdot 6 \mathrm{H}_{2} \mathrm{O}\right]$ para a condensação de $\beta$-ceto ésteres com aminas primárias, secundárias, benzílicas e aromáticas em excelentes rendimentos (Esquema 5). Como essa reação é sensível a efeitos estéricos, quando são empregados aminas impedidas e/ou $\beta$-ceto ésteres que possuem substituintes na posição $\alpha$, é necessário que se aqueça o sistema na temperatura de refluxo $\left(40{ }^{\circ} \mathrm{C}\right)$. O catalisador empregado nesse sistema também é bastante reativo, estável ao ar, de baixo custo e pode ser recuperado. Exemplos ilustrativos podem ser vistos no Esquema 5.

Khosropour et al. ${ }^{18}$ relataram a síntese de $\beta$-enaminonas em bons rendimentos (63-98\%), empregando trifluoracetado de bismuto(III) em quantidades catalíticas, conforme exemplificado no Esquema 6. São utilizadas aminas alifáticas e aromáticas e o 

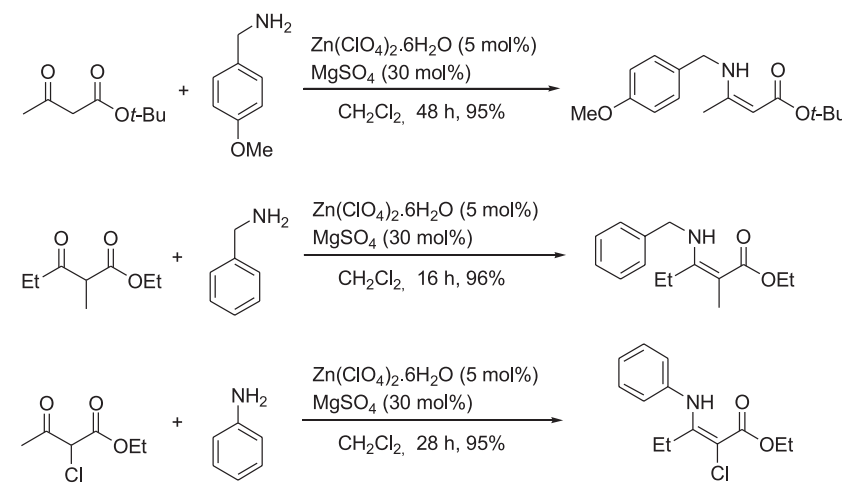

Esquema 5.

catalisador é recuperado e reutilizado. Esse procedimento é bastante interessante, porque além de utilizar água como solvente, emprega um catalisador que é estável em meio aquoso, de baixo custo e pouco tóxico.
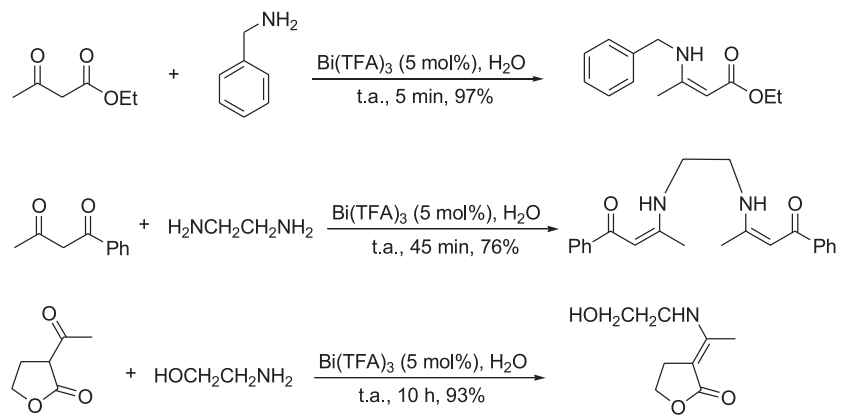

Esquema 6.

Uma série de imidoilbenzotriazóis foi preparada por Katritzky et al. ${ }^{19}$, reagindo amidas secundárias com 1 -cloro-1- $H$-benzotriazol na presença de trifenilfosfina. Os imidoilbenzotriazóis preparados foram utilizados na síntese de $\beta$-enamino cetonas através da substituição da benzotriazoíla com silil enolatos (Esquema 7).

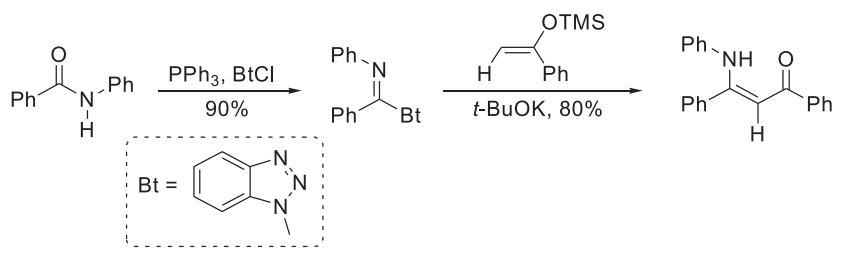

Esquema 7.

Reddy et al. ${ }^{20}$ sintetizaram uma série de $\beta$-enaminonas através de cicloadição $[3+2]$ de enonas ativadas por ácido de Lewis com alquil azidas. O intermediário 1,2,3-triazolínico sofre rearranjo in situ, através de uma contração de anel, fornecendo as enaminonas correspondentes, como se vê no exemplo do Esquema 8. Vários ácidos de Lewis, como $\mathrm{BF}_{3} . \mathrm{Et}_{2} \mathrm{O}, \mathrm{TFA}, \mathrm{TiCl}_{4}, \mathrm{MeAlCl}_{2}, \mathrm{Yb}(\mathrm{OTf})_{3} \mathrm{e}$ ácido trífico, foram utilizados, sendo que os melhores resultados foram obtidos empregando-se TMSOTf.

Tendo em vista a importância sintética de $\alpha$-iodoenaminonas, Kim et al. ${ }^{21}$ prepararam uma série desses compostos usando iodo e trietilamina em excelentes rendimentos (Esquema 9).

$\beta$-Trifluorometil enaminonas foram preparadas estereosseletivamente através da reação de amidas com acetileto trifluorometilado de lítio ${ }^{22}$ e também através da adição de aminas a $\beta$-clorovinil- $\beta$ trifluorometil cetonas ${ }^{23}$

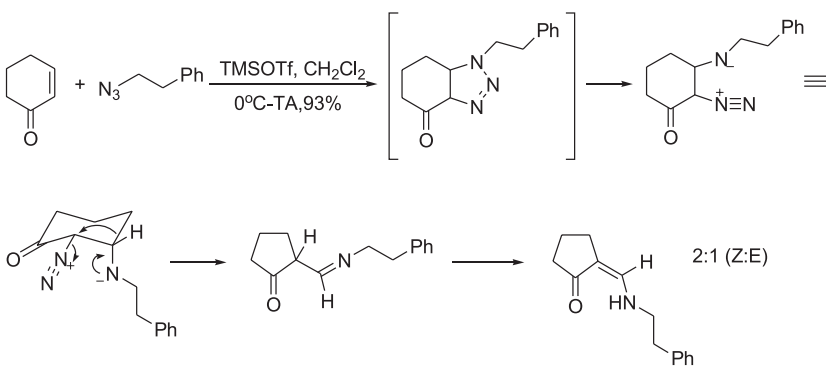

Esquema 8.
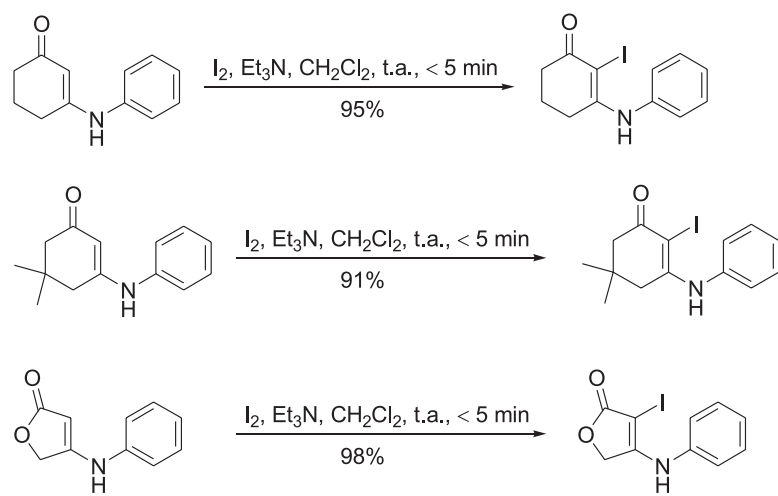

Esquema 9.

\section{APLICAÇÕES SINTÉTICAS}

A procura de métodos cada vez mais eficientes de preparação de enaminonas está relacionada com a variedade de reações em que estes compostos podem ser empregados, principalmente na síntese de heterociclos dos mais variados tipos. Um grande número de fármacos e agroquímicos biologicamente ativos é de heterociclos, bem como inúmeros aditivos e modificadores usados na indústria de cosméticos, armazenagem e plásticos ${ }^{24,25}$.

Em recente revisão, Stanovnik e Svete ${ }^{4}$ relatam a preparação e a aplicação de 3-(dimetilamino)propenoatos e análogos na síntese de furanos, piranos, pirróis, pirazóis, piridinas, pirimidinas e seus análogos policíclicos. Em outra revisão, Svete apresenta resultados específicos sobre a síntese de pirazóis funcionalizados ${ }^{26}$.

Usando enaminonas derivadas da cânfora, o mesmo grupo de pesquisa $^{27}$ descreve a síntese de pirazóis, através da ciclocondensação com hidrazina mostrada no Esquema 10.

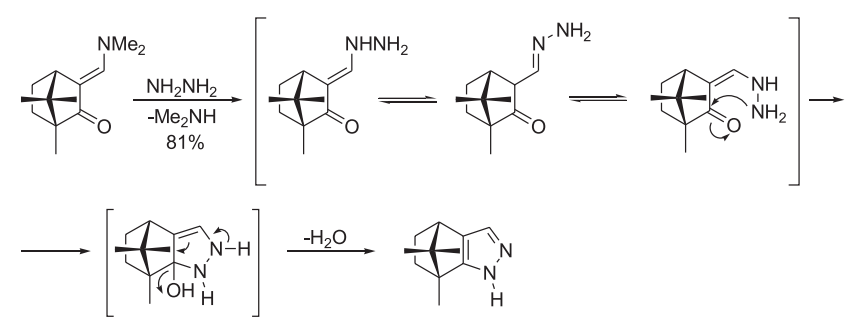

Esquema 10.

A partir de $\beta$-enaminonas sililadas, que foram obtidas por clivagem redutiva de sililmetilisoxazóis, Calle et al. ${ }^{28}$ sintetizaram sililpirazóis. Após a hidrogenação catalítica dos isoxazóis sililados, reagiram-se as enaminonas formadas com hidrazinas para obtenção do anel pirazólico sililado. Alguns exemplos poder ser visualizados no Esquema 11. 


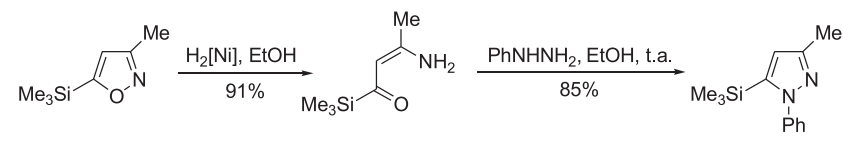

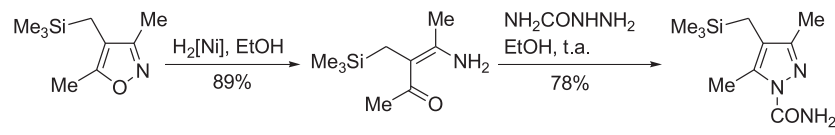

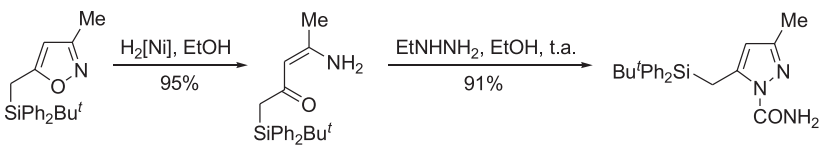

Esquema 11.

Estratégia semelhante de ciclocondensação foi utilizada para obtenção de pirazóis trifluorometilados, pela reação de trifluorometil enaminonas com hidrazinas monossubstituídas ${ }^{29}$.

Pirazóis opticamente puros foram obtidos a partir de lactamas ativadas derivadas do ácido itacônico ${ }^{30}$ (Esquema 12). A enaminona formada a partir da tiolactama sofre um ataque binucleofílico da hidrazina, levando ao pirazol correspondente.

Estratégia similar foi utilizada pelo mesmo grupo para obter $\omega$ heterociclos $\alpha$-amino ácidos com diferentes padrões de substituição $0^{31}$.

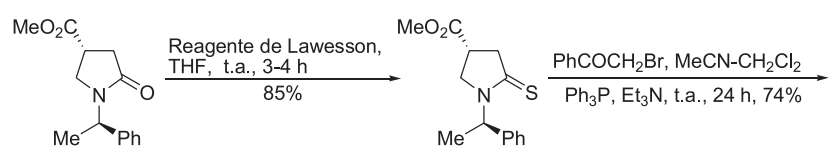

$\mathrm{MeO}_{2} \mathrm{C}$

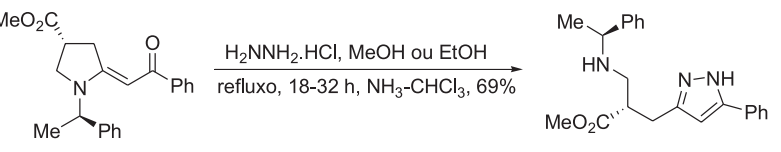

1. $\mathrm{HCOOH}$ ou cicloexeno, $10 \% \mathrm{Pd}-\mathrm{C}$

refluxo, $6-18 \mathrm{~h}$

2. $(\mathrm{Boc})_{2} \mathrm{O}, \mathrm{Et}_{3} \mathrm{~N}, \mathrm{CH}_{2} \mathrm{Cl}_{2}, 0^{\circ} \mathrm{C}, 2-3 \mathrm{~h}$

3. $\mathrm{MeOH}-\mathrm{HCl}, \mathrm{O} \rightarrow 25^{\circ} \mathrm{C}, 12 \mathrm{~h}$

$94 \%$

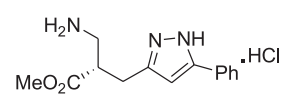

$6 \mathrm{~N} \mathrm{HCl}$, refluxo, 2-4 $\mathrm{h}$ $90 \%$<smiles>NC[C](Cc1cc(-c2ccccc2)[nH]n1)C(=O)O</smiles>

Esquema 12.

A preparação de 1,2,3-triazóis foi relatada por Melo et al. ${ }^{32}$ utilizando a 5,7-dinitro-3-diazo-isatina e mesilazida como compostos de transferência do grupo diazo para enaminonas, como mostrado no Esquema 13.

Demir et al..$^{33}$ sintetizaram pirróis funcionalizados através da condensação de compostos 2-propinil-1,3-dicarbonílicos com aminas,<smiles>CCOC(=O)/C=C(/C)NC(C)(C)C</smiles><smiles>CNC(C)=CC(C)=O</smiles>

Esquema 13. catalisada por ácido trifluoracético. Inicialmente ocorre a aminação do composto carbonílico, seguida da ciclização regiosseletiva (5exo-dig) da enaminona e posterior aromatização com formação do anel pirrólico. Um exemplo é mostrado no Esquema 14.<smiles>C#CCC(C(C)=O)C(=O)OCC</smiles><smiles>Nc1ccccc1</smiles>

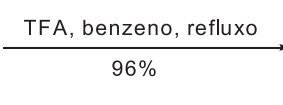<smiles>CCOC(=O)c1cc(C)n(-c2ccccc2)c1C</smiles>

Esquema 14.

Em trabalho anterior, o mesmo grupo ${ }^{34}$ preparou pirróis 1,2,3,5tetrassubstituídos a partir de enaminonas (Esquema 15).

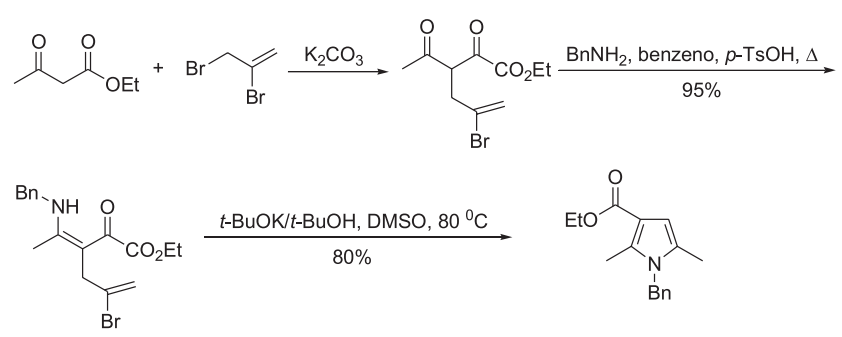

Esquema 15.

Pirróis funcionalizados também foram obtidos pela propargilação de enaminonas, seguida de uma hidroaminação intramolecular catalisada por nitrato de $\operatorname{prata}^{35}$ (Esquema 16).

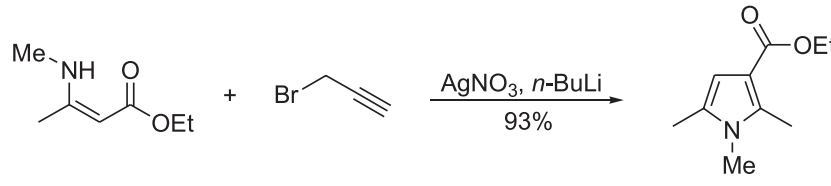<smiles>C#CCBr</smiles><smiles>C#CCBr</smiles>

Esquema 16.

Singh et al. ${ }^{36}$ descrevem a obtenção de 2-pirrolidinonas, 1,5diidro-2-pirrolonas e pirrolidinas $N$-substituídas (Esquema 17). $\mathrm{O}$ aduto de Baylis-Hillman é obtido através da reação do 3-isoxazolcarbaldeído com acrilato de metila. A hidrogenólise do aduto de Baylis-Hillman leva a uma enaminona, que sofre ciclização intramolecular fornecendo a pirrolidina correspondente.

Os estudos da hidrogenação catalítica dos adutos de BaylisHilman foram apresentados em trabalho anterior ${ }^{37}$.

Nosso grupo de pesquisa, nos últimos anos, tem utilizado a ciclofuncionalização para obtenção de heterociclos oxigenados ${ }^{38,39}$ e nitrogenados ${ }^{40,41}$. Em recente trabalho ${ }^{42}$, aplicamos a iodociclofuncionalização em $\beta$-enamino ésteres para obtenção de tetraidropiridinas que, após reação de desidroiodação, forneceram ciclopentanos trissubstituídos (Esquema 18)

Dando continuidade aos trabalhos de iodociclofuncionalização, 


$$
\begin{aligned}
& \text { 每 } \\
& \text { 等 } \\
& \underbrace{}_{\mathrm{NHBn}} \longrightarrow \underbrace{\mathrm{NH}_{2}}_{\mathrm{BNN}_{\mathrm{H}}^{\oplus}}-\mathrm{CO}_{2} \mathrm{Me} \longrightarrow \\
& \longrightarrow
\end{aligned}
$$

Esquema 17.

preparamos 4-fenil-1,4,5,6-tetraidropiridinas a partir dos $\beta$ enamino ésteres correspondentes ${ }^{43}$.

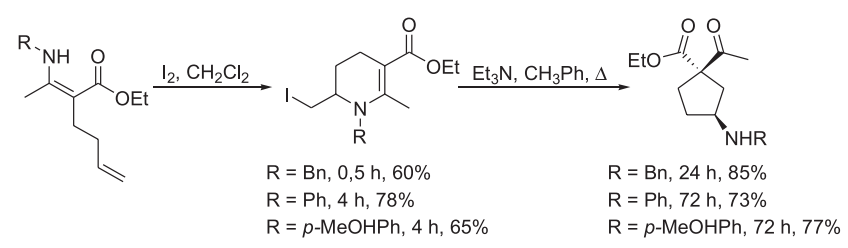

Esquema 18.

White e Ihle ${ }^{44}$ relatam seus estudos de fotociclização [2+2] intramolecular seguida de uma retro-Mannich de enaminonas a partir da triptamina e $\beta$-fenetilaminas, gerando $\Delta^{1}$-pirrolinas (Esquema 19).<smiles>CCOC(=O)C=C(OCC)C(=O)OCC</smiles><smiles>CCOC(=O)C(=CNCCc1cn(Br)c2ccccc12)OCC</smiles>

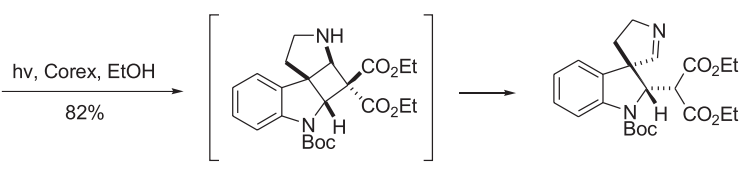

Esquema 19.

Uma série de 6-arilpiridinas 2,3-dissubstituídas foi preparada por condensação de enaminonas, usando K-10 como suporte ácido sólido ${ }^{45}$. A reação das enamino cetonas com acetoacetato de etila forneceu o derivado piridínico em bons rendimentos, como pode ser visualizado no Esquema 20.

Davis et $a l .{ }^{46}$ desenvolveram uma metodologia para a preparação de derivados piperidínicos 2,4,5-trissubstituídos a partir de $\delta$ amino $\beta$-ceto enaminonas. Esse protocolo é baseado em uma seqüência de cinco etapas "one-pot", que envolve a formação da enaminona com dimetilformamida dimetilacetal, hidrólise, adição de Michael intramolecular seguida de uma eliminação tipo retroMichael e proteção. Após hidrogenação, foi obtido o intermediário enólico chave, o que consiste de uma síntese formal da pseudodistomina B, uma vez que o mesmo intermediário foi obtido por Ma e $\mathrm{Sun}^{47}$ para a síntese assimétrica do triacetato da pseudodistomina B (Esquema 21).

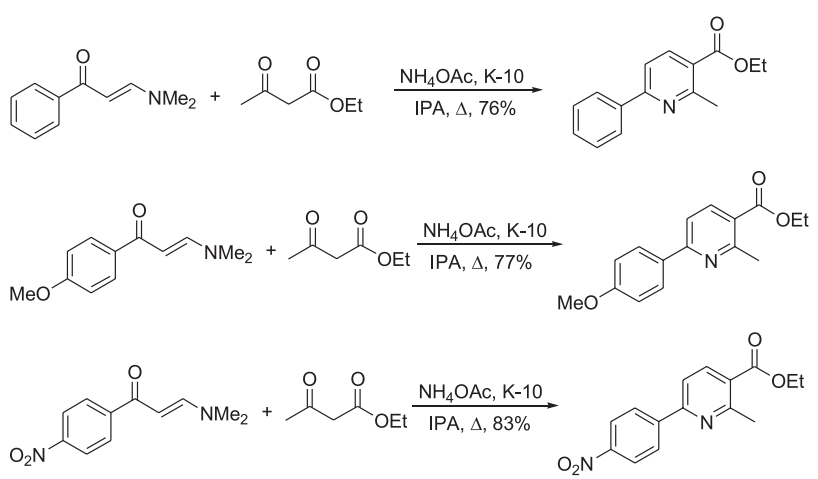

Esquema 20

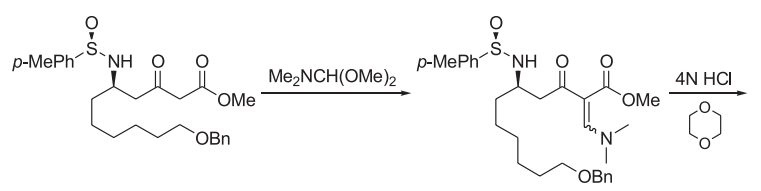
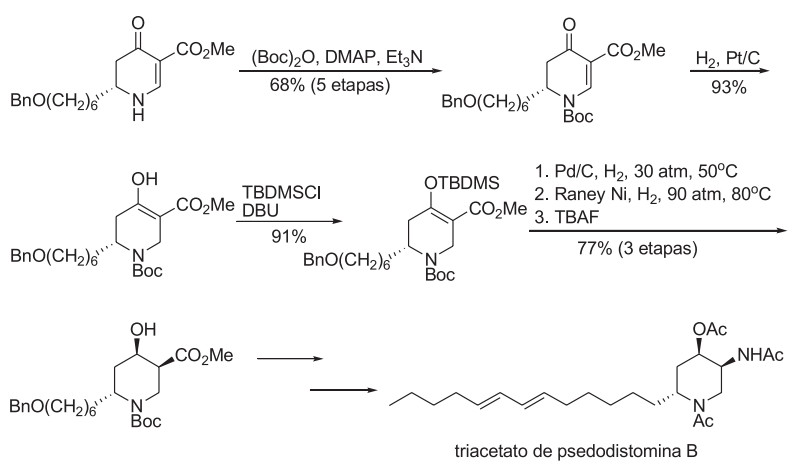

Esquema 21.

Através da reação de ciclização de enaminonas com cloretos de acró́la foram obtidos derivados de piridinonas ${ }^{48}$ (Esquema 22). A reação de aza-anelação não foi bem sucedida quando foram utilizados outros eletrófilos, como anidrido malêico, dicarboxilato de dimetilacetileno e propiolato de etila.
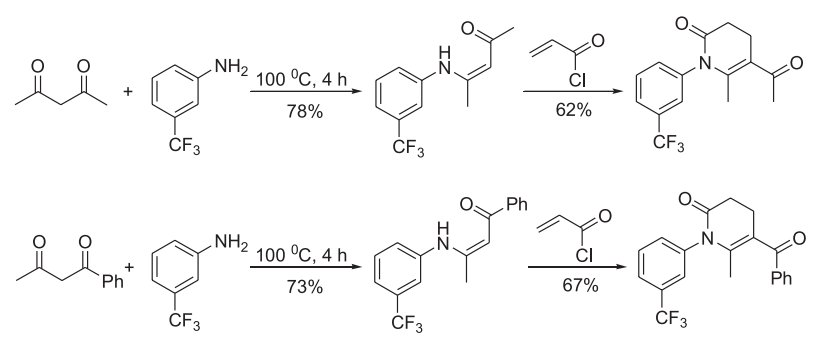

Esquema 22.

A aza-anelação intramolecular de $\beta$-enaminonas também foi utilizada por Cunha et al. ${ }^{49}$ para obtenção de 2-piridonas (Esquema 23). A reação de enaminonas acíclicas com o derivado metoximetilênico do ácido de Meldrum forneceu $N$-adutos e/ou $C$-adutos das enaminonas em bons rendimentos, sendo o $C$-aduto utilizado como precursor de 2-piridonas.

As enaminonas como materiais de partida na síntese de diversos heterociclos vêm sendo sistematicamente exploradas pelo grupo de Elnagdi ${ }^{50}$. Um exemplo pode ser visualizado no Esquerma 24, onde se mostra a preparação da piridona pela reação da enaminona com o cianoacetato de etila, na presença de hidreto de sódio ${ }^{51}$. 
<smiles>CCOCCNC(C)=C(C=C1C(=O)OC(C)(C)OC1=O)C(C)=O</smiles><smiles>CCOC(=O)Cn1c(C)c(C(C)=O)cc(C(=O)O)c1=O</smiles>

Esquema 23.

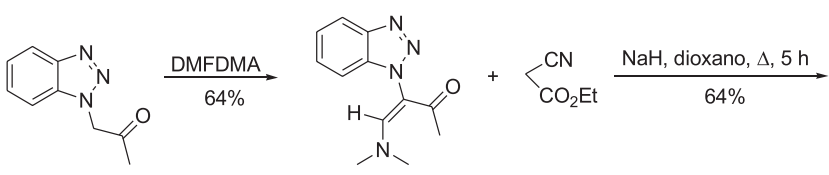<smiles>CCOC(=O)c1c(C)c(-n2nnc3ccccc32)c[nH]c1=O</smiles>

Esquema 24.

Kidemet et al..$^{52}$ desenvolveram um método para a preparação de $N$-fenil-2-aminopirimidinas através da ciclocondensação de $N$ fenilguanidinas com enaminonas na presença de DBU, sem uso de solvente. Um exemplo de formação do anel pirimidínico pode ser visualizado no Esquema 25.<smiles>COc1cc(NC(N)=N[NH2+][O-])cc(OC)c1OC</smiles>

Esquema 25.

Tetraidropirimidinas foram obtidas através do refluxo de enaminonas em metanol com diaminas e formaldeído ${ }^{53}$ (Esquema 26). Em trabalho anterior, o mesmo grupo ${ }^{54}$ utilizou aminas primárias para obtenção de tetraidropirimidinas.

Muitos derivados quinolínicos podem ser preparados a partir de enaminonas. Si et al. ${ }^{55}$ prepararam enaminonas através da adição de aminas à tripla ligação de mesilatos de $\alpha, \alpha, \alpha$-triclorometilpropargila, que são usados na preparação de 2-fenil-4-diclorometilquinolinas (Esquema 27).

Outra série de derivados quinolínicos e indolizidínicos foi sin-<smiles>O=C(C1=CN(c2ccccc2)CN(CCN2C=C(C(=O)c3ccccc3)CN(c3ccccc3)C2)C1)c1ccccc1</smiles>

Esquema 26.
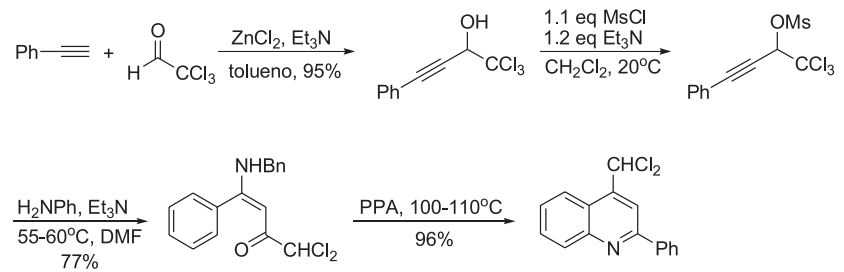

Esquema 27.

tetizada a partir de hidróxi enaminonas via oxidação catalisada por paládio ${ }^{56}$.

Back et al. ${ }^{57}$ sintetizaram os alcalóides quinolizidínicos (-)-lasubina II e ( \pm )-mirtina, através da redução estereosseletiva e dessulfonilação de enaminonas cíclicas, como pode ser observado na preparação da (-)-lasubina II (Esquema 28).

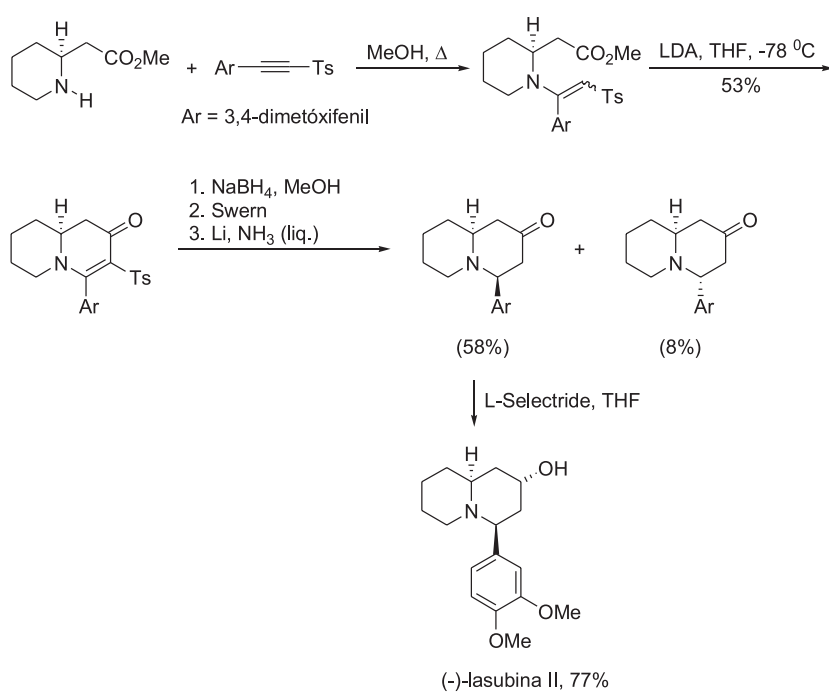

Esquema 28.

Michael et al. ${ }^{58}$ prepararam uma série de esqueletos indolizidínicos pela ciclização de enaminonas, através de hidrólise ácida e ativação do ácido carboxílico como anidrido (Esquema 29). Em trabalho anterior, Michael et al. ${ }^{59}$ utilizaram $\beta$-sulfonil enaminonas para a síntese de indolizidinonas.

Sorensen e Pombo-Villar ${ }^{60}$ utilizaram a reação de Heck intramolecular de $\alpha$-haloenaminonas para obtenção de indolonas e carbazolonas (Esquema 30). A enaminona foi formada através da condensação da 2-iodoanilina com a cicloexano-1,3-diona, que após ciclização catalisada por paládio, forneceu a carbazolona em $95 \%$ de rendimento.

As enaminonas são bastante utilizadas na preparação de $\gamma$-amino álcoois, que são unidades estruturais presentes em diversos compostos com propriedades farmacológicas e produtos naturais biologicamente ativos. $\mathrm{O}$ método mais utilizado para se obter $\gamma$-amino álcoois é a redução estereosseletiva de $\beta$-enamino cetonas, usan- 


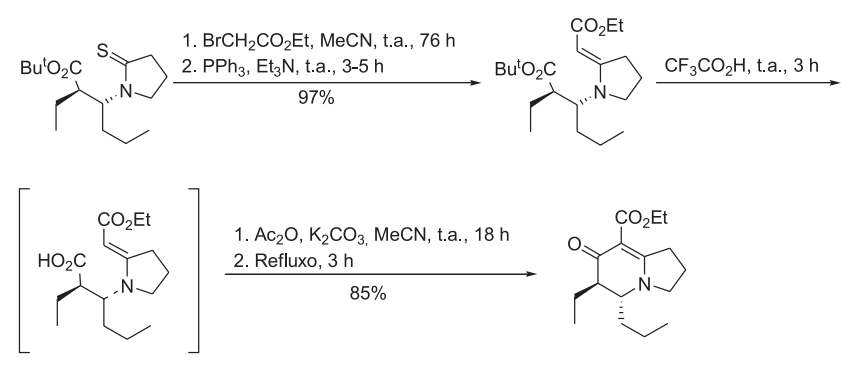

Esquema 29.
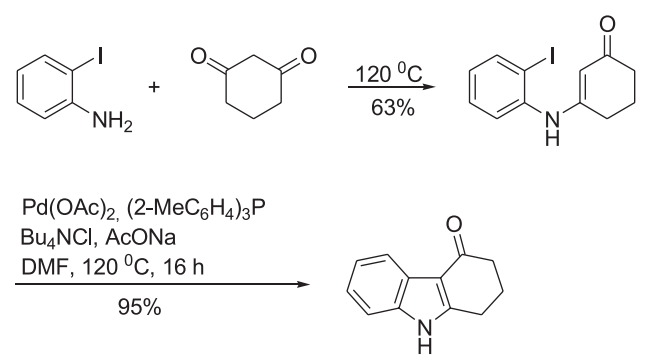

Esquema 30.

do-se agentes redutores como $\mathrm{LiBH}_{4}$ na presença de $\mathrm{CeCl}_{3}{ }^{61} \mathrm{e}$ $\mathrm{NaBH}_{4} / \mathrm{AcOH}^{62}$.

Um procedimento de redução diastereosseletiva da dupla ligação da enaminona, para obtenção de derivados quirais de $\beta$-amino ácidos, é através da hidrogenação catalítica. Ikemoto et al. ${ }^{63}$ utilizaram $\mathrm{PtO}_{2}$ como catalisador e a fenilglicina amida (PGA) como auxiliar quiral na hidrogenação diastereosseletiva de uma série de enaminonas (Esquema 31)

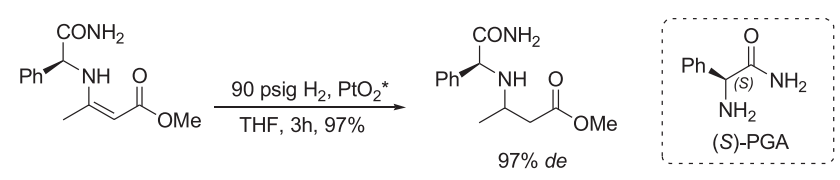

*superfície lavada com $\mathrm{AcOH}$

Esquema 31.

Outras reações onde as enaminonas são usadas como intermediários sintéticos são encontradas na literatura com uma variedade de aplicações ${ }^{64-69}$.

\section{CONCLUSÕES}

De maneira geral, no desenvolvimento de novos métodos para a preparação de $\beta$-enaminonas, observa-se uma preocupação em diminuir o tempo reacional e aumentar os rendimentos, além de utilizar catalisadores estáveis, acessíveis e menos prejudiciais, empregar sistemas que tenham um menor impacto ambiental e evitar o uso de solventes.

O aperfeiçoamento das metodologias de preparação de enaminonas, assim como sua grande versatilidade reacional, têm contribuído para que essa classe de compostos se torne cada vez mais utilizada como intermediários sintéticos, principalmente na síntese de heterociclos.

\section{AGRADECIMENTOS}

À FAPESP pelo contínuo apoio financeiro e pela bolsa de doutorado (E. R. S. Gonçalo) e ao CNPq pela bolsa de produtividade (H. M. C. Ferraz).

\section{REFERÊNCIAS}

1. Ferraz, H. M. C.; Pereira, F. L. C.; Quim. Nova 2004, 27, 89.

2. Negri, G.; Kascheres, C.; Kascheres, A. J.; J. Heterocycl. Chem. 2004, 41, 461.

3. Elassar, A.-Z. A.; El-Khair, A. A.; Tetrahedron 2003, 59, 8463.

4. Stanovnik, B.; Svete, J.; Chem. Rev. 2004, 104, 2433.

5. Svete, J.; Monats. Chem. 2004, 135, 629.

6. Greenhill, J. V.; Chem. Soc. Rev. 1977, 6, 277.

7. Lue, P.; Greenhill, J. V.; Adv. Heterocycl. Chem. 1997, 67, 207.

8. Granik, V. G.; Russ. Chem. Rev. (Engl. Trans.) 1984, 53, 383.

9. Ferreira, V. F.; de Souza, M. C. B. V.; Cunha, A. C.; Pereira, L. O. R.; Ferreira, M. L. G.; Org. Prep. Proced. Int. 2001, 33, 411.

10. Michael, J. P.; Koning, C. B.; Gravestock, D.; Hosken, G. D.; Howard, A. S.; Krause, R. W. M.; Parsons, A. S.; Pelly, S. C.; Stanbury, T, V.; Pure Appl. Chem. 1999, 71, 979.

11. Stefani, H. A.; Costa, I. M.; Silva, D. O.; Synthesis 2000, 1526.

12. Katritzky, A. R.; Ghiviriga, I.; Oniciu, D. C.; O'Ferrall, R. A. M.; Walsh, S. M.; J. Chem. Soc., Perkin Trans. 2 1997, 2605.

13. Gholap, A. R.; Chakor, N. S.; Daniel, T.; Lahoti, R. J.; Srinivasan, K. V.; J. Mol. Catal. A: Chem. 2006, 245, 37.

14. Braibante, M. E. F.; Braibante, H. T. S.; Morel, A. F.; Costa, C. C.; Lima, M. G.; J. Braz. Chem. Soc. 2006, 17, 184.

15. Dalpozzo, R.; De Nino, A.; Nardi, M.; Russo, B.; Procopio, A.; Synthesis 2006, 1127.

16. Kobayashi, S.; Sugiura, M.; Kitagawa, H.; Lam, W. W.-L.; Chem. Rev. 2002, 102, 2227.

17. Bartoli, G.; Bosco, M.; Locatelli, M.; Marcantoni, E.; Melchiorre, P.; Sambri, L.; Synlett 2004, 239.

18. Khosropour, A. R.; Khodaei, M. M.; Kookhazadeh, M.; Tetrahedron Lett. 2004, 45, 1725.

19. Katritzky, A. R.; Hayden, A. E.; Kirichenko, K.; Pelphrey, P.; Ji, Y.; J. Org. Chem. 2004, 69, 5108

20. Reddy, D. S.; Judd, W. R.; Aubé, J.; Org. Lett. 2003, 5, 3899.

21. Kim, J. M.; Na, J. E.; Kim, J. N.; Tetrahedron Lett. 2003, 44, 6317.

22. Jeong, I. H.; Jeon, S. L.; Kim, S. M.; Kim, B. T.; J. Fluorine Chem. 2004, $125,1629$.

23. Zouaoui, E.; Rezgui, F.; El Gaïed, M. M.; J. Chem. Res. 2004, 811.

24. Katritzky, A. R.; Chem. Rev. 2004, 104, 2125.

25. Melo, J. O. F.; Donnici, C. L.; Augusti, R.; Ferreira, V. F.; Souza, M. C. B.; Ferreira, M. L. G.; Cunha, A. C.; Quim. Nova 2006, 29, 569.

26. Svete, J.; Arkivoc 2006, vii, 35

27. Groselj, U.; Bevk, D.; Jakse, R.; Recnik, S.; Meden, A.; Stanovnik, B.; Svete, J.; Tetrahedron 2005, 61, 3991.

28. Calle, M.; Calvo, L. A.; González-Ortega, A.; González-Nogal, A. M.; Tetrahedron 2006, 62, 611

29. Touzot, A.; Soufyane, M.; Berber, H.; Toupet, L.; Mirand, C.; J. Fluorine Chem. 2004, 125, 1299.

30. Singh, R. K.; Sinha, N.; Jain, S.; Salman, M.; Naqvi, F.; Anand, N.; Tetrahedron 2005, 61, 8868.

31. Singh, R. K.; Sinha, N.; Jain, S.; Salman, M.; Naqvi, F.; Anand, N.; Synthesis 2005, 2765.

32. Melo, J. O. F.; Ratton, P. M.; Augusti, R.; Donnici, C. L.; Synth. Commun. 2004, 34, 369 .

33. Demir, A. S.; Aybey, A.; Kayalar, M.; Arkivoc 2005, xv, 105.

34. Demir, A. S.; Akhmedov, I. M.; Sesenoglu, O.; Tetrahedron 2002, 58, 9793.

35. Robinson, R. S.; Dovey, M. C.; Gravestock, D.; Tetrahedron Lett. 2004, $45,6787$.

36. Singh, V.; Saxena, R.; Batra, S.; J. Org. Chem. 2005, 70, 353.

37. Saxena, R.; Singh, V.; Batra, S.; Tetrahedron 2004, 60, 10311.

38. Ferraz, H. M. C.; Sano, M. K.; Nunes, M. R. S.; Bianco, G.; J. Org. Chem. 2002, 67, 4122.

39. Ferraz, H. M. C.; Sano, M. K.; Bombonato, F. I.; Nunes, M. R. S.; J. Braz. Chem. Soc. 2005, 16, 884 .

40. Ferraz, H. M. C.; Pereira, F. L. C.; Leite, F. S.; Nunes, M. R. S.; PayretArrúa, M. E.; Tetrahedron 1999, 55, 10915.

41. Ferraz, H. M. C.; Payret-Arrúa, M. E.; Brandt, C. A.; J. Org. Chem. 1995, 60, 7357.

42. Ferraz, H. M. C.; Pereira, F. L. C.; Gonçalo, E. R. S.; Santos, L. S.; Eberlin, M. N.; J. Org. Chem. 2005, 70, 110.

43. Ferraz, H. M. C.; Gonçalo, E. R. S.; Resumos da $26^{a}$ Reunião Anual da Sociedade Brasileira de Química, Poços de Caldas, Brasil, 2003; Ferraz, H. M. C.; Gonçalo, E. R. S.; Resumos do $11^{\circ}$ Brazilian Meeting on Organic Synthesis, Canela, Brasil, 2005.

44. White, J. D.; Ihle, D. C.; Org. Lett. 2006, 8, 1081.

45. Reddy, G. J.; Latha, D.; Thirupathaiah, C.; Rao, K. S.; Tetrahedron Lett. $\mathbf{2 0 0 5}, 46,301$ 
46. Davis, F. A.; Zhang, J.; Li, Y.; Xu, H.; DeBrosse, C.; J. Org. Chem. 2005, $70,5413$.

47. Ma, D.; Sun, H.; J. Org. Chem. 2000, 65, 6009

48. Ali, A. A.; Winzenberg, K. N.; Aust. J. Chem. 2005, 58, 870.

49. Cunha, S.; Silva, V. C.; Napolitano, H. B.; Lariucci, C.; Vencato, I.; J. Braz. Chem. Soc. 2003, 14, 107.

50. Al-Saleh, B.; El-Apasery, M. A.; Abdel-Aziz, R. S.; Elnagdi, M. H.; J. Heterocycl. Chem. 2005, 42, 563; Abdallah, S. O.; Metwally, N. H.; Anwar, H. F.; Elnagdi, M. H.; J. Heterocycl. Chem. 2005, 42, 781; Abdelkhalik, M. M.; Eltoukhy, A. M.; Agamy, S. M.; Elnagdi, M. H.; J. Heterocycl. Chem. 2004, 41, 431; Almazroa, S.; Elnagdi, M. H.; El-Din, A. M. S.; J. Heterocycl. Chem. 2004, 41, 267; Ghozlan, S. A. S.; Abdelhamid, I. A.; Gaber, H.; Elnagdi, M. H.; J. Chem. Res. 2004, 789; Al- Mousawi, S.; Abdelkhalik, M. M.; John, E.; Elnagdi, M. H.; J. Heterocycl. Chem. 2003, $40,689$.

51. Al-Saleh, B.; Behbehani, H.; El-Apasery, M. A.; Elnagdi, M. H.; J. Chem. Res. 2004, 575.

52. Kidemet, D.; Elenkov, I.; Prgomet, V.; Synlett 2005, 2531.

53. Dutta, M. C.; Chanda, K.; Vishwakarma, J. N.; J. Heterocycl. Chem. 2005, $42,121$.

54. Chanda, K.; Dutta, M. C.; Karim, E.; Vishwakarma, J. N.; J. Heterocycl. Chem. 2004, 41, 627.

55. Si, Y.-G.; Guo, S.-P.; Wang, W.-J.; Jiang, B.; J. Org. Chem. 2005, 70, 1494.

56. Pita, B.; Masaguer, C. F.; Raviña, E.; Tetrahedron Lett. 2002, 43, 7929.

57. Back, T. G.; Hamilton M. D.; Lim, V. J. J.; Parvez, M.; J. Org. Chem. 2005, $70,967$.
58. Michael, J. P.; Koning, C. B.; Westhuyzen, C. W.; Org. Biomol. Chem. 2005 , 3, 836.

59. Michael, J. P.; Koning, C. B.; Malefetse, T. J.; Yillah, I.; Org. Biomol. Chem. 2004, 2, 3510 .

60. Sorensen, U.; Pombo-Villar, E.; Helv. Chim. Acta 2004, 87, 82.

61. Bartoli, G.; Cupone, G.; Dalpozzo, R.; De Nino, A.; Maiuolo, L.; Procopio, A.; Tagarelli, A.; Tetrahedron Lett. 2002, 43, 7441.

62. Harris, M. I. N. C.; Braga, A. C. H.; J. Braz. Chem. Soc. 2004, 15, 971.

63. Ikemoto, N.; Tellers, D. M.; Dreher S. D.; Liu, J.; Huang, A.; Rivera, N. R.; Njolito, E.; Hsiao, Y.; McWilliams, J. M.; Armstrong, J. D. III; Sun, Y.; Mathre, D. J.; Grabowski, E. J. J.; Tillyer, R. D.; J. Am. Chem. Soc. 2004, 126, 3048.

64. Cunha, S.; Rodovalho, W.; Azevedo, N. R.; Mendonça, M. O.; Lariucci, C.; Vencato, I.; J. Braz. Chem. Soc. 2002, 13, 629.

65. Reznikov, V. A.; Roshchupkina, G. I.; Mazhukin, D. G.; Petrov, P. A.; Popov, S. A.; Fokin, S. V.; Romanenko, G. V.; Rybalova, T. V.; Gatilov, Y. V.; Shvedenkov, Y. G.; Irtegova, I. G.; Shundrin, L. A.; Ovcharenko, V. I.; Eur. J. Org. Chem. Soc. 2004, 749.

66. Simunek, P.; Péskova, M.; Bertolasi, V.; Machacek, V.; Lycka, A.; Eur. J. Org. Chem. 2005, 8130.

67. Valla, A.; Valla, B.; Carties, D.; Le Guillou, R.; Labia, R.; Potier, P.; Tetrahedron Lett. 2005, 46, 6671.

68. Al-Omran, F.; El-Khair, A.; J. Heterocycl. Chem. 2005, 42, 307.

69. Ming, L.; Shuwen, W.; Lirong, W.; Huazheng, Y.; Xiuli, Z.; J. Heterocycl. Chem. 2005, 42, 925. 